\title{
Concentração de retinol em bebidas lácteas achocolatadas enriquecidas em comparação às quantidades declaradas no rótulo
}

\author{
Penha Patrícia Cabral Ribeiro ${ }^{1}$, Mayara Santa Rosa Lima ${ }^{1}$, \\ Andressa Fernanda de Lima Beserra ${ }^{2}$, Heryka Myrna Maia Ramalho ${ }^{3}$, \\ Evellyn Câmara Grilo ${ }^{1}$, Priscila Nunes Costa ${ }^{1}$, Roberto Dimenstein ${ }^{4}$
}

\begin{abstract}
A bebida láctea achocolatada é um produto lácteo amplamente consumido e que pode ser enriquecido com nutrientes essenciais, como forma de reforçar o seu valor nutricional e prevenir ou corrigir carências específicas na população, especialmente em crianças. Um desses nutrientes é a vitamina A, cuja deficiência é considerada um problema de saúde pública. O objetivo deste estudo foi quantificar o retinol presente em bebidas lácteas achocolatadas Ultra High Temperature - UHT enriquecidas e verificar a compatibilidade com os valores especificados nos rótulos. Para tanto, foram selecionadas aleatoriamente três marcas desses produtos ( $\mathrm{A}, \mathrm{B}$ e $\mathrm{C}$ ), analisadas através de Cromatografia Líquida de Alta Eficiência (CLAE). A concentração de retinol é dada em $\mu \mathrm{g} / \mathrm{dL}$ e os valores expressos em média e desviopadrão. Os resultados mostraram que as marcas A e $\mathrm{C}$ apresentaram concentração de retinol condizente com a descrita nos rótulos $(70,5 \pm 6,8 \mu \mathrm{g} / \mathrm{dL}$ e $73,5 \pm 8,2 \mu \mathrm{g} / \mathrm{dL})$. Entretanto, a marca B apresentou concentração muito baixa $(9,0 \pm 3,2 \mu \mathrm{g} / \mathrm{dL})$, conflitante com a declarada $(75,0 \mu \mathrm{g} / \mathrm{dL})$. Tal situação evidencia a ausência de suplementação no achocolatado da marca B, fazendo-se necessária uma maior fiscalização nesses produtos, a fim de garantir que as informações presentes nos rótulos sejam confiáveis.
\end{abstract}

Palavras-chave: produtos lácteos, suplementação alimentar, vitamina A.

\section{Retinol concentration in milk chocolate beverages compared to the amounts declared on the label}

Milk chocolate beverage is a dairy product widely consumed and can be enriched with essential nutrients to enhance its nutritional value and prevent or correct specific deficiencies in the population, especially in children. One of these nutrients is vitamin A, whose deficiency is considered a public health problem. This study aimed to quantify the retinol present in fortified achocolatadas Ultra High Temperature - UHT milk chocolate beverage and check its compatibility with the values specified on the label. For this purpose, were randomly selected three brands of these products (A, B and C), analyzed by High Performance Liquid Chromatography (HPLC). The concentration of retinol is given in $\mu \mathrm{g} / \mathrm{dL}$ and values expressed as mean and standard deviation. The results show that brands $\mathrm{A}$ and $\mathrm{C}$ had retinol concentration consistent with that described on the label $(70.5 \pm 6.8 \mu \mathrm{g} / \mathrm{dL}$ and $73.5 \pm 8.2 \mu \mathrm{g} / \mathrm{dL})$. However, the brand B had a very low concentration $(9.0 \pm 3.2 \mu \mathrm{g} / \mathrm{dL})$, in conflict with the declared $(75.0 \mu \mathrm{g} / \mathrm{dL})$. This situation

\footnotetext{
${ }^{1}$ Graduanda em Nutrição, Universidade Federal do Rio Grande do Norte (UFRN), Centro de Ciências da Saúde, Departamento de Nutrição.

${ }^{2}$ Nutricionista formada pela Universidade Potiguar (UNP), Natal (RN), Brasil - Laureate International Universities.

${ }^{3}$ Docente, UNP, Natal (RN), Brasil - Laureate International Universities.

${ }^{4}$ Professor $(\mathrm{PhD})$, UFRN, Centro de Biociências, Departamento de Bioquímica, Laboratório de Bioquímica de Alimentos e Nutrição. Correspondência: Departamento de Bioquímica, Centro de Biociências, UFRN. Av. Senador Salgado Filho, no 3000. Bairro: Lagoa Nova, Natal/RN, CEP: 59072-970. Fone: 55 (84) 3215-3416, ramal 205. Fax: 55 (84)33422812.E-mail: rdimenstein@gmail.com.
} 
highlights the lack of supplementation in chocolate brand B. Thus, greater supervision of supplementation of these products is necessary to avoid insufficient intake of vitamin A and to ensure the reliability of the information contained on labels.

Key-words: dairy products, supplementary feeding, vitamin A.

\section{INTRODUÇÃO}

Bebida láctea é o produto resultante da mistura do leite e soro de leite adicionado ou não de produtos ou substâncias alimentícias, gordura vegetal, leites fermentados, fermentos lácteos selecionados e outros produtos lácteos [1]. Essa bebida é consumida por vários grupos da população, devido às suas características sensoriais e nutricionais, conveniência e praticidade [2].

A bebida láctea achocolatada se destaca entre o público infantil, tendo um papel importante no crescimento e desenvolvimento das crianças, por ser derivada do leite. Esse alimento contém proteínas de alto valor biológico e nutrientes essenciais, como cálcio, fósforo, biotina, vitamina $\mathrm{D}, \mathrm{B}_{2}$ e $\mathrm{A}$ [3]. Esta última, também chamada de retinol, é indispensável para visão, imunidade, crescimento e diferenciação celular, além de atuar na síntese de glicoproteínas e na reprodução [4].

Assim como outros produtos líquidos e semilíquidos, a bebida láctea achocolatada pode passar por um processo térmico denominado de UHT (Ultra High Temperature), que ocorre por meio do seu aquecimento, durante 2 a 4 segundos, a $130^{\circ} \mathrm{C}-150^{\circ} \mathrm{C}$, mediante um processo térmico de fluxo contínuo, com imediata redução de temperatura para menos de $32^{\circ} \mathrm{C}$. Em seguida, o produto é envasado em condições assépticas, em embalagens estéreis e hermeticamente fechadas ${ }^{[1]}$, podendo ser conservado por até seis meses em temperatura ambiente (ainda fechado).

Antecedendo esse processo, o produto pode ser enriquecido/fortificado com nutrientes essenciais [3], com o intuito de compensar as perdas que ocorrem durante o processamento e armazenamento [5], reforçar o seu valor nutritivo e/ou prevenir ou corrigir deficiências nutricionais existentes na população [6].
Um dos nutrientes comumente adicionados a leites e bebidas lácteas é a vitamina $\mathrm{A}$, cuja deficiência responde por uma parcela importante dos índices de morbidade e mortalidade infantil e atinge áreas além do Norte e Nordeste do Brasil, sendo independente do mapa econômico do país [7]. Em nível mundial, a Deficiência de Vitamina A (DVA) é considerada um problema de saúde pública ${ }^{[8]}$.

Desta forma, o leite e seus derivados são considerados importantes veículos de nutrientes, por meio de fortificação, por serem alimentos de consumo massivo, sendo de grande relevância no combate a carências específicas [5].

Uma vez enriquecidos, esses produtos devem conter essa informação no rótulo, assim como a composição nutricional em relação à percentagem da Ingestão Diária Recomendada (\% da IDR) de forma quantitativa por $100 \mathrm{~mL}$ e, ainda, opcionalmente, por porção consumida [6]. Esses dados devem ser seguros e confiáveis, pois é através deles que o consumidor conhece o produto [9]. Portanto, existe a necessidade de se fiscalizar tais informações, o que inclui a análise da composição química, a fim de dosar as quantidades dos nutrientes suplementados nesses alimentos [10,11].

Diante do papel essencial da vitamina A no organismo, da significante prevalência de sua deficiência e da importância da fidedignidade das informações contidas nos rótulos de alimentos suplementados com nutrientes essenciais, este trabalho foi desenvolvido buscando quantificar a vitamina A presente em bebidas lácteas achocolatadas UHT enriquecidas e verificar a compatibilidade com os valores especificados nos rótulos.

\section{METODOLOGIA}

Foram selecionadas aleatoriamente três marcas de bebida láctea achocolatada suplementada com vitamina A comercializadas em Natal (Rio Grande do Norte). Os produtos adquiridos não 
apresentavam deformações ou danos aparentes nas embalagens e possuíam o menor tempo de fabricação possível.

Para as análises, cada marca foi representada por seis caixas, de três lotes diferentes. Anteriormente à extração, as caixas foram submetidas à temperatura de $40^{\circ} \mathrm{C}$ em banho-maria durante 10 minutos, objetivando a dissolução da gordura na amostra, para evitar perdas decorrentes da retenção de vitamina A nas paredes internas da embalagem.

Para extração da vitamina, o conteúdo de duas caixas do mesmo lote foi colocado em um béquer de $500 \mathrm{~mL}$ protegido da luz, sendo homogeneizado durante 10 minutos em agitador magnético Fanem ${ }^{\circledR}$, modelo 257 (São Paulo, Brasil). A partir desse procedimento, obteve-se uma única amostra representativa do lote, da qual foram retiradas duas alíquotas de $1 \mathrm{~mL}$, que foram transferidas para tubos de polipropileno protegidos da luminosidade. Esse procedimento foi repetido com os demais lotes.

A extração foi realizada a partir de uma modificação do método de Giuliano et al. [12]. Para tanto, foram adicionados $1 \mathrm{~mL}$ de álcool etílico $95 \%$ (Vetec, Rio de Janeiro, Brasil) e $1 \mathrm{~mL}$ de hidróxido de potássio 50\% (Vetec, Rio de Janeiro, Brasil) aos tubos contendo $1 \mathrm{~mL}$ de achocolatado, para possibilitar a desnaturação protéica e a hidrólise alcalina, respectivamente. Os tubos foram agitados por 1 minuto e mantidos em banho-maria a $60^{\circ} \mathrm{C}$ durante 1 hora, sendo agitados a cada 10 minutos.

Como reagente extrativo, utilizou-se o hexano (Quimex, São Paulo, Brasil), adicionando-se $2 \mathrm{~mL}$ deste a cada tubo, que foram posteriormente agitados durante 1 minuto e centrifugados por 10 minutos, após os quais o extrato hexânico sobrenadante foi transferido para outro tubo. Tal procedimento foi feito por mais duas vezes, totalizando $6 \mathrm{~mL}$ de extrato, que foram agitados para retirada de uma alíquota de $2 \mathrm{~mL}$. Esta foi evaporada em banho-maria a $37^{\circ} \mathrm{C}$ e o extrato seco obtido foi redissolvido em $500 \mu$ de etanol absoluto (Merck, São Paulo, Brasil) em grau de pureza para Cromatografia Líquida de Alta Eficiência (CLAE).

$\mathrm{Na}$ análise bioquímica, a concentração de retinol das amostras foi determinada por CLAE em cromatógrafo constituído de bomba LC-20 AT
Shimadzu, acoplado a um detector SPD-20A Shimadzu UV-VIS, coluna Shim-pack CLC-ODS (M) $4,6 \mathrm{~mm} \times 15 \mathrm{~cm}$ e computador com programa LC solution $\quad\left(\right.$ Shimadzu Corporation $\left.{ }^{\circledR}\right)$ para processamento dos dados. A evolução do cromatograma foi feita nas seguintes condições: metanol 100\% e fluxo $1,0 \mathrm{~mL} / \mathrm{min}$. A identificação e quantificação do retinol nas amostras foi estabelecida por meio da comparação com o tempo de retenção e área do respectivo padrão. A concentração do padrão foi confirmada pelo coeficiente de extinção específico $(\varepsilon 1 \%, 1 \mathrm{~cm}=1850) \mathrm{em}$ etanol absoluto (Merck, São Paulo, Brasil) e comprimento de onda de $325 \mathrm{~nm}{ }^{[13]}$.

A concentração de retinol é dada em $\mu \mathrm{g} / \mathrm{dL}$ e os valores expressos em média e desvio-padrão.

\section{RESULTADOS}

A Figura 1 mostra o perfil cromatográfico a $325 \mathrm{~nm}$ do padrão de retinol com concentração de 26,19 ng/20 $\mu \mathrm{L}$ e de uma amostra de bebida láctea achocolatada enriquecida com vitamina $\mathrm{A}$. O pico do retinol aparece isolado, com tempo de retenção de 3,0 minutos.

Foi observado que uma das marcas de bebida láctea achocolatada (B) possuía, em média, 9,0 $\pm 3,2$ $\mu \mathrm{g} / \mathrm{dL}$ de retinol, enquanto a quantidade de vitamina A declarada no rótulo era de 75,0 $\mu \mathrm{g} / \mathrm{dL}$ (Figura 2). Assim, o valor obtido foi equivalente a apenas $12 \%$ do expresso na rotulagem.

As demais marcas possuíam quantidades do micronutriente condizentes com as citadas na embalagem, mantendo-se dentro da margem de variação estabelecida pela legislação $(20 \%)$ [14]. O achocolatado A obteve uma média de 70,5 $\pm 6,8$ $\mu \mathrm{g} / \mathrm{dL}$, próxima ao valor declarado no rótulo $(75,0$ $\mu \mathrm{g} / \mathrm{dL})$. Já o achocolatado C possuía, em média, 73,5 $\pm 8,2 \mu \mathrm{g} / \mathrm{dL}$ de retinol, também semelhante ao declarado $(67,5 \mu \mathrm{g} / \mathrm{dL})$ (Figura 2).

\section{DISCUSSÃO}

Nos últimos anos o consumidor vem apresentando preocupações em relação à sua dieta, o que se reflete no hábito de ler as informações nutricionais nos rótulos dos produtos, que devem, portanto, ser corretas e completas [2], tendo em vista que essa descrição representa um parâmetro de 

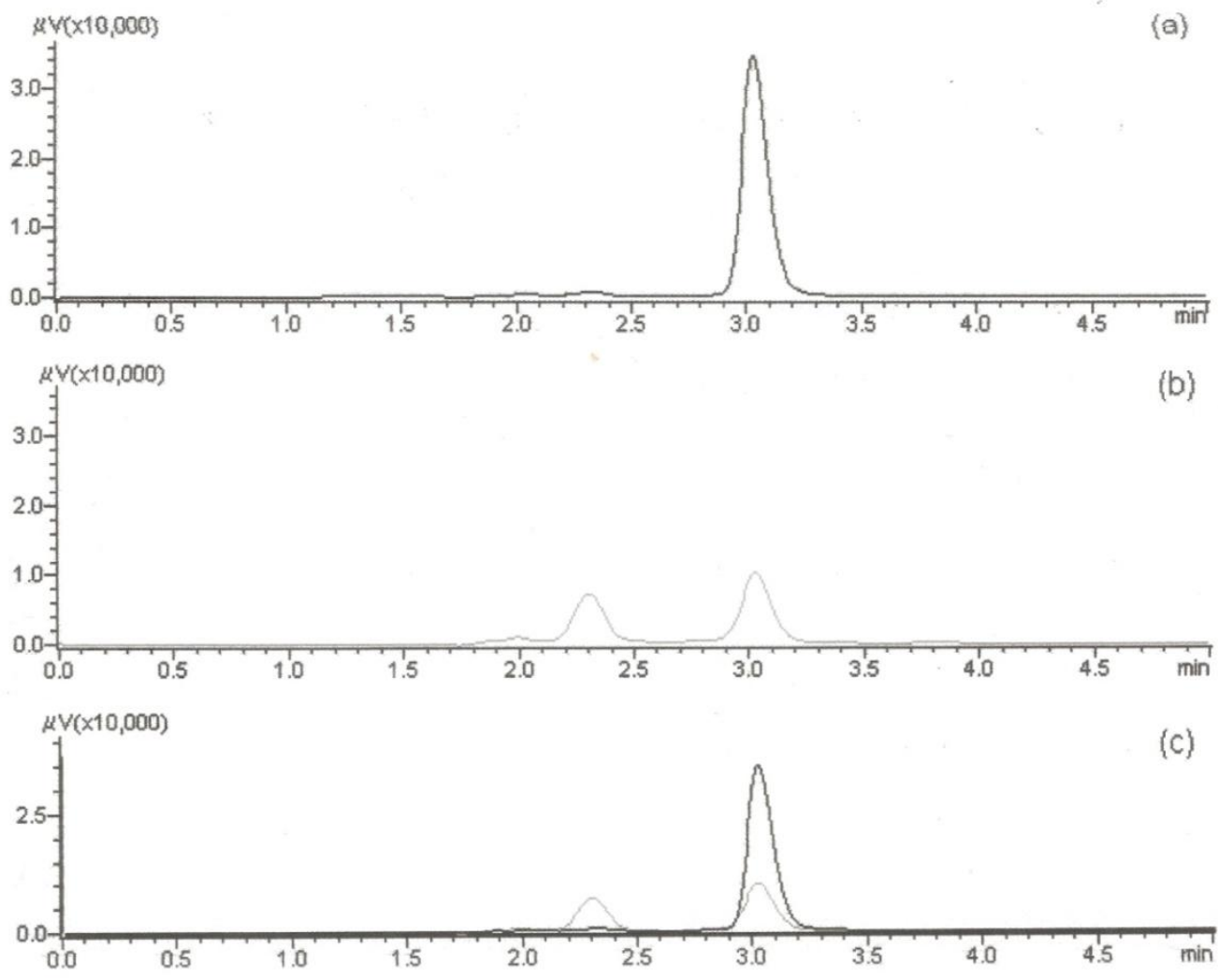

Figura 1. Picos de retinol a $325 \mathrm{~nm}$ do padrão de retinol (26,19 ng) (a), de uma amostra de bebida láctea achocolatada enriquecida (b) e sobreposição desses (c).

comparação ${ }^{[10]}$ que permite a escolha de opções alimentares mais saudáveis.

Educação alimentar, suplementação através de medicamentos e enriquecimento de alimentos têm sido utilizadas para equilibrar e garantir uma alimentação saudável. Nesse contexto, a fortificação/enriquecimento de alimentos em nível industrial é um dos melhores processos para o domínio das carências nutricionais de micronutrientes da população em todo o mundo [15], sendo realizada através da suplementação de alimentos com expressivo consumo, como é o caso do leite e seus derivados $[5,16]$.

A eficiência da fortificação para corrigir, erradicar ou prevenir doenças específicas causadas por deficiências de nutrientes, como a DVA, é amplamente reconhecida [15]. Entretanto, Garcia \& Penteado [17] atentam para o fato de que o enriquecimento de alimentos com vitaminas requer cuidados tecnológicos, para que a sua retenção durante o processamento e a vida de prateleira seja assegurada. Assim, na fortificação de alimentos com esses micronutrientes, devido à sua instabilidade, existe a necessidade de sobredosá-los, para assegurar os níveis recomendados pela legislação e declarados na embalagem.

No presente estudo, foi observado que uma das marcas de bebida láctea enriquecida analisada possuía concentração de vitamina $\mathrm{A}$ menor do que a encontrada em leites de vaca achocolatados não suplementados que, segundo a Tabela Brasileira de Composição de Alimentos [18], é de $39 \mu \mathrm{g} / \mathrm{dL}$. Esse dado, aliado ao fato de ter sido encontrada uma média de retinol 88\% menor do que a descrita no rótulo da bebida láctea em questão demonstra que, pode não ter havido fortificação com vitamina A nos lotes analisados. Portanto, a bebida láctea da marca B está em desacordo com a legislação, que determina que o nutriente suplementado deva estar presente numa 
concentração que não implique ingestão insignificante [6].

Para ser considerado enriquecido ou fortificado, um alimento líquido deve conter acima de 15\% da Ingestão Diária de Referência (IDR) do nutriente em $100 \mathrm{~mL}$ [1]. A IDR para vitamina A é de $600 \mu \mathrm{g}$ para adultos; porém, considerando que a maioria das bebidas lácteas enriquecidas é produzida tendo como público alvo o infantil, tem-se que a recomendação para crianças de 1 a 3 anos é de $400 \mu \mathrm{g}$, aumentando para $450 \mu \mathrm{g}$ entre 4 e 6 anos e para 500 $\mu \mathrm{g}$ de 7 a 10 anos [19]. Portanto, considerando esses dados, a menor quantidade presente em um produto desse tipo e que caracterizaria uma bebida láctea enriquecida com vitamina A seria de $60 \mu \mathrm{g} / \mathrm{dL}(15 \%$ da menor IDR). Assim, a bebida láctea da marca B não pode ser considerada enriquecida, levando em conta os lotes analisados.
A deficiência de vitamina A pode levar a xeroftalmia, cegueira e morte [20]. Isso é preocupante, uma vez que se os produtos ditos enriquecidos com esta vitamina não apresentarem seriedade e segurança em sua fortificação, o consumo de produtos fortificados objetivando alcançar melhor aporte nutricional, possivelmente, não será alcançado, podendo implicar em problemas como a ingestão reduzida do micronutriente [?].

Segundo Abrantes [9], alguns produtos intitulados como fortificados possuem quantidades de nutrientes equivalentes aos produtos similares não fortificados, o que pode aumentar o preço de um produto que não proporciona benefício real. Isso demonstra que a suplementação de alimentos com nutrientes é, muitas vezes, uma simples estratégia de marketing, que não leva em conta a verdadeira necessidade dos consumidores ${ }^{[11]}$.

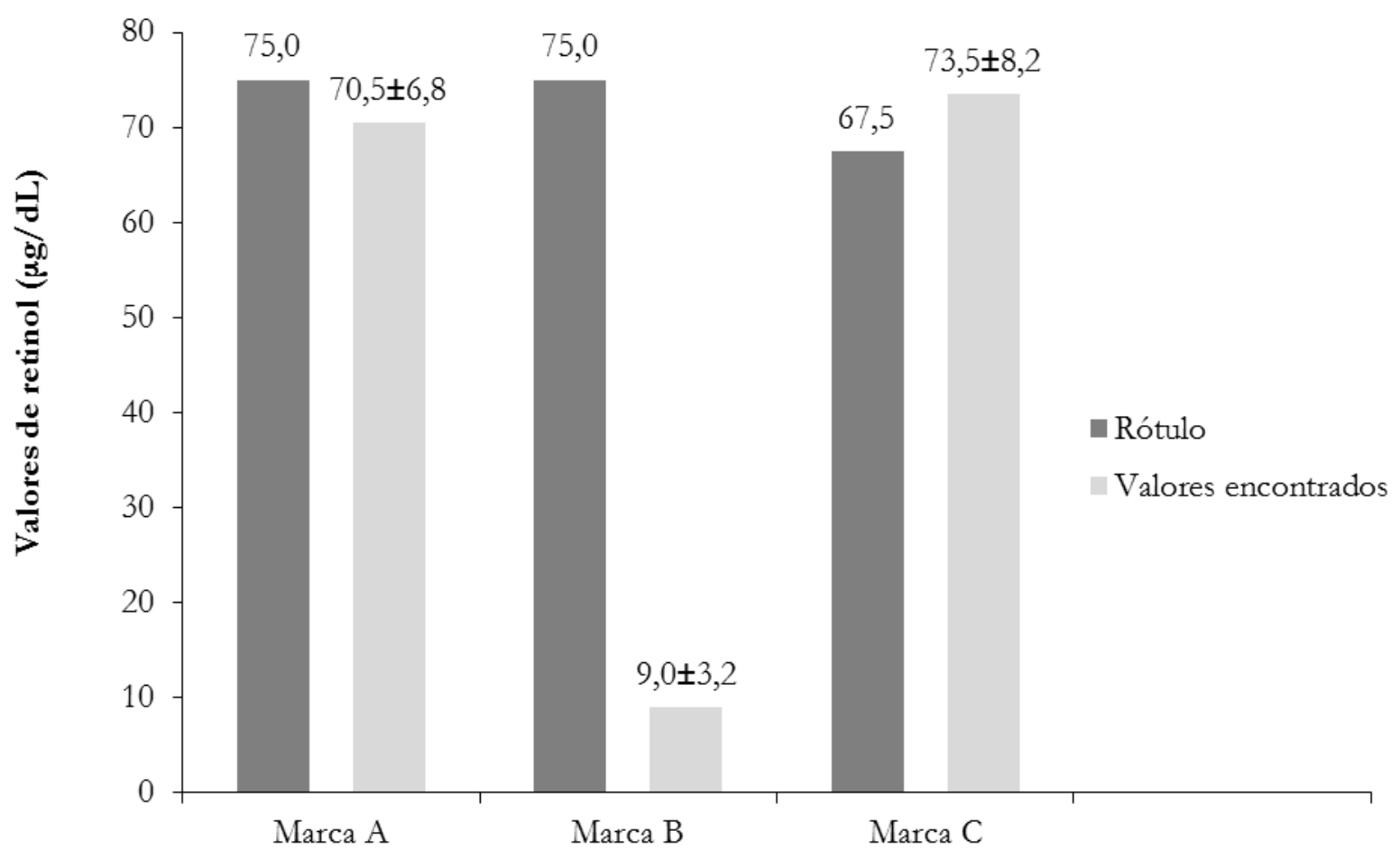

Figura 2. Valores de retinol $(\mu \mathrm{g} / \mathrm{dL})$ em bebidas lácteas achocolatadas UHT enriquecidas. 


\section{CONCLUSÕES}

Uma das marcas de bebida láctea enriquecida possuía média de retinol muito abaixo da expressa no rótulo, apontando para a existência de uma suplementação inadequada desses produtos, o que pode colocar em risco a correta ingestão do micronutriente pela população.

Portanto, para que os produtos estejam de acordo com as especificações do fabricante, se faz necessário o enriquecimento com quantidades adequadas para garantir a eficácia da suplementação, além de maior fiscalização quanto ao enriquecimento dos produtos e à confiabilidade das informações nutricionais presentes nos rótulos, a fim de evitar possíveis danos ao consumidor.

\section{REFERÊNCIAS}

[1] Brasil. Ministério da Agricultura, Pecuária e Abastecimento. Instrução Normativa no 16, de 23 de agosto de 2005. Aprova o regulamento técnico de identidade e qualidade de bebidas lácteas. Diário Oficial da União, Brasilia, DF, 24 ago. 2005. Seção 1, p. 7.

[2] Eduardo MF, Silva LSC. Achocolatados: análise química. Rev Bras Cienc Farm. 2004;40(3):405-12

[3] Philippi ST. Pirâmide dos alimentos - Fundamentos básicos da nutrição. 1o ed. São Paulo: Manole; 2008.

[4] Gallagher ML. Vitaminas. In: Mahan LK, Escott-stump S, editores. Krause: Alimentos, nutrição e dietoterapia. 11으 ed. São Paulo: Roca; 2005.

[5] Abranches MV, Della Lucia CM, Sartori MA, PinheiroSant'Ana HM. Perdas de vitaminas em leite e produtos lácteos e possíveis medidas de controle. Alim Nutr. 2008;19(2):207-17.

[6] Brasil. Agência Nacional de Vigilância Sanitária do Ministério da Saúde. Portaria no 31, de 13 de janeiro de 1998. Regulamento técnico para fixação de identidade e qualidade de alimentos adicionados de nutrientes essenciais. Diário Oficial da União, Brasília, 16 jan. 1998. Seção 1, p. 4.

[7] Ramalho RA, Flores H, Saunders C. Hipovitaminose A no Brasil: um problema de saúde pública. Rev Panam Salud Pública. 2002;12(2):117-23.
[8] Milagres RCRM, Nunes LC, Pinheiro-Sant'Ana HM. A deficiência de vitamina A em crianças no Brasil e no mundo. Ciênc Saúde Colet. 2007;12(5):1253-66.

[9] Abrantes VRS. Rotulagem de alimentos: análise em fórmulas infantis, leites em pó e alimentos em pó à base de soja comercializados no varejo do município do Rio de Janeiro/RJ [dissertação]. Seropédica: Universidade Federal Rural do Rio de Janeiro; 2007. 141 p.

[10] Câmara MCC, Marinho CLC, Guilam MC, Braga AMCB. A produção acadêmica sobre a rotulagem de alimentos no Brasil. Rev Panam Salud Pública. 2008;23(1):52-8.

[11] Paixão JA, Stamford TLM. Vitaminas lipossolúveis em alimentos - uma abordagem analítica. Quim Nova. 2004; 27(1):96-105.

[12] Giuliano AR, Neilson EM, Kelly BE, Canfield LM. Simultaneous quantitation and separation of carotenoids and retinol in human milk by high-performance liquid chromatography. Methods Enzymol. 1992;213:391-9.

[13] Nierenberg DW, Nann SL. A method for determining concentrations of retinol, tocopherol and five carotenoids in human plasma and tissue samples. Am J Clin Nutr. 1992;56(2):417-26.

[14] Brasil. Agência Nacional de Vigilância Sanitária do Ministério da Saúde do Brasil. Resolução RDC no 360, de 23 de dezembro de 2003. Aprova o regulamento técnico sobre rotulagem nutricional de alimentos embalados. Diário Oficial da União, Brasília, 26 dez. 2003. Seção 1, p. 33.

[15] Zancul MS. Fortificação de alimentos com ferro e vitamina A. Medicina, Ribeirão Preto. 2004;37:45-50.

[16] Lima JA, Catharino RR, Godoy HT. Ácido fólico em leite e bebida láctea enriquecidos - estudo da vida-deprateleira. Ciênc Tecnol Aliment. 2004;24(1):82-7.

[17] Garcia T, Penteado MVC. Qualidade de balas de gelatina adicionadas de vitaminas A, C e E. Ciênc Tecnol Aliment. 2005;25(4):743-9.

[18] Tabela Brasileira de Composição de Alimentos. 2o ed. Campinas: Nepa-Unicamp; 2004.

[19] Brasil. Agência Nacional de Vigilância Sanitária do Ministério da Saúde do Brasil. Resolução RDC no 269, de 22 de setembro de 2005. Aprova o regulamento técnico sobre a ingestão diária recomendada (IDR) de proteína, vitaminas e 
minerais. Diário Oficial da União, Brasilia, 23 set. 2005.

Seção 1, p. 372.

[20] Almeida-Muradian LB, Penteado MVC. Vitamina A. In: Penteado MVC. Vitaminas: aspectos nutricionais, bioquímicos, clínicos e analíticos. 1ำ ed. São Paulo: Ed. Manole; 2003. p. 55-72. 